ISSN 1855-3966 (printed edn.), ISSN 1855-3974 (electronic edn.)

ARS MATHEMATICA CONTEMPORANEA 15 (2018) 347-354

https://doi.org/10.26493/1855-3974.1459.11d

(Also available at http://amc-journal.eu)

\title{
Mirrors of reflections of regular maps
}

\author{
Adnan Melekoğlu * \\ Department of Mathematics, Faculty of Arts and Sciences, \\ Adnan Menderes University, 09010 Aydın, Turkey
}

Received 4 August 2017, accepted 8 October 2017, published online 27 June 2018

\begin{abstract}
A regular map $\mathcal{M}$ is an embedding of a finite connected graph into a compact surface $S$ such that its automorphism group $\operatorname{Aut}^{+}(\mathcal{M})$ acts transitively on the directed edges. A reflection of $\mathcal{M}$ fixes a number of simple closed geodesics on $S$, which are called mirrors. In this paper, we prove two theorems which enable us to calculate the total number of mirrors fixed by the reflections of a regular map and the lengths of these mirrors. Furthermore, by applying these theorems to Hurwitz maps, we obtain some interesting results. In particular, we find an upper bound for the number of mirrors on Hurwitz surfaces.
\end{abstract}

Keywords: Riemann surface, regular map, Hurwitz map, reflection, mirror.

Math. Subj. Class.: 05C10, 30F10

\section{Introduction}

Let $S$ be a compact Riemann surface of genus $g$. It is known that $S$ can be expressed in the form $\mathbb{U} / \Lambda$, where $\mathbb{U}$ is the Riemann sphere $\Sigma$, the Euclidean plane $\mathbb{C}$, or the hyperbolic plane $\mathbb{H}$, depending on whether $g$ is 0,1 or $>1$, respectively, and $\Lambda$ is a discrete group of isometries of $\mathbb{U}$. A conformal or anti-conformal homeomorphism $f: S \rightarrow S$ is called an automorphism of $S$. If $S$ admits an anti-conformal involution $r: S \rightarrow S$, then it is called symmetric and $r$ is called a symmetry of $S$. The fixed-point set of $r$ is either empty, or consists of disjoint simple closed geodesics on $S$. These geodesics are called the mirrors of $r$ and their number cannot exceed $g+1$ by a classical theorem of Harnack [10]. All automorphisms of $S$ form a group under composition and it is denoted by $\operatorname{Aut}^{ \pm}(S)$. The subgroup of $\mathrm{Aut}^{ \pm}(S)$ consisting of orientation-preserving automorphisms is denoted by $\operatorname{Aut}^{+}(S)$.

${ }^{*}$ The author would like to thank the referees for their valuable comments and suggestions. The author would also like to thank David Singerman for suggesting the alternative proof of Theorem 3.1 described in Remark 3.4.

E-mail address: amelekoglu@adu.edu.tr (Adnan Melekoğlu)

(a) (i) This work is licensed under http://creativecommons.org/licenses/by/3.0/ 
Let $T$ be a triangle in $\mathbb{U}$, with angles $\pi / 2, \pi / m$ and $\pi / n$, where $m$ and $n$ are integers greater than one and $\frac{1}{m}+\frac{1}{n}$ is greater than, equal to or less than $\frac{1}{2}$ depending on whether $\mathbb{U}$ is $\Sigma, \mathbb{C}$ or $\mathbb{H}$, respectively. Such a triangle is said to be a $(2, m, n)$-triangle. Let $\Gamma$ be the group generated by the rotations about the corners of $T$. Then it is called the ordinary triangle group $\Gamma[2, m, n]$ and it has a presentation

$$
\left\langle x, y, z \mid x^{2}=y^{m}=z^{n}=x y z=1\right\rangle .
$$

If $\Gamma$ is the group generated by the reflections in the sides of $T$, then it is called the extended triangle group $\Gamma(2, m, n)$, which has a presentation

$$
\left\langle a, b, c \mid a^{2}=b^{2}=c^{2}=(a b)^{2}=(b c)^{m}=(c a)^{n}=1\right\rangle .
$$

A map $\mathcal{M}$ on $S$ is an embedding of a finite connected graph $G$ into $S$ such that the interior of each face (a component of $S \backslash G$ ) is homeomorphic to an open disc. The genus of $\mathcal{M}$ is defined to be the genus of $S$. A directed edge of $\mathcal{M}$ is called a dart and $\mathcal{M}$ is said to be of type $\{m, n\}$ if every face of $\mathcal{M}$ has $m$ sides and $n$ darts meet at every vertex. An automorphism of $S$ that leaves $\mathcal{M}$ invariant and preserves incidence is called an automorphism of $\mathcal{M}$. All automorphisms of $\mathcal{M}$ form a group under composition and this group is denoted by $\operatorname{Aut}^{ \pm}(\mathcal{M})$. The subgroup of $\mathrm{Aut}^{ \pm}(\mathcal{M})$ consisting of orientationpreserving automorphisms is denoted by $\operatorname{Aut}^{+}(\mathcal{M})$. If $\mathrm{Aut}^{+}(\mathcal{M})$ is transitive on the darts, then $\mathcal{M}$ is called regular. It is clear that if $\mathcal{M}$ is regular, then the number of darts is equal to $\left|\operatorname{Aut}^{+}(\mathcal{M})\right|$ and $\mathcal{M}$ has $\left|\operatorname{Aut}^{+}(\mathcal{M})\right| / 2$ edges, $\left|\operatorname{Aut}^{+}(\mathcal{M})\right| / m$ faces and $\left|\operatorname{Aut}^{+}(\mathcal{M})\right| / n$ vertices.

If $\mathcal{M}$ is a regular map of type $\{m, n\}$ and $S=\mathbb{U} / \Lambda$ is the underlying Riemann surface, then by [12], $\Lambda$ is normal in the ordinary triangle group $\Gamma[2, m, n]$. If $\Lambda$ is also normal in the extended triangle group $\Gamma(2, m, n)$, then $\mathcal{M}$ is called reflexible. In that case $\mathcal{M}$ admits an anti-conformal involution $r$, which is a symmetry of $S$ with fixed-points, called a reflection of $\mathcal{M}$.

In this paper, we prove two theorems which enable us to calculate the total number of mirrors fixed by the reflections of a regular map and the lengths of these mirrors. Furthermore, we use these theorems to obtain an upper bound for the total number of mirrors in Hurwitz maps.

Throughout this paper, we assume that the maps we deal with are regular and reflexible.

\section{Patterns and mirror automorphisms}

Let $\mathcal{M}$ be a regular map of type $\{m, n\}$ on a compact Riemann surface $S$ of genus $g$. By joining the centers of the faces of $\mathcal{M}$ to the midpoints of the neighboring edges and vertices by geodesic arcs, we can divide $S$ into $\left|\operatorname{Aut}^{ \pm}(\mathcal{M})\right|(2, m, n)$-triangles. If $T$ is one of these triangles, then the group $\operatorname{Aut}^{ \pm}(\mathcal{M})$ can be generated by the reflections in the sides of $T$ and it has a presentation of the form

$$
\left\langle A, B, C \mid A^{2}=B^{2}=C^{2}=(A B)^{2}=(B C)^{m}=(C A)^{n}=\cdots=1\right\rangle .
$$

Similarly, the group $\mathrm{Aut}^{+}(\mathcal{M})$ can be generated by the rotations about the corners of $T$ and it has a presentation of the form

$$
\left\langle X, Y, Z \mid X^{2}=Y^{m}=Z^{n}=X Y Z=\cdots=1\right\rangle .
$$


Note that if $g=0$, then the groups $\operatorname{Aut}^{ \pm}(\mathcal{M})$ and $\operatorname{Aut}^{+}(\mathcal{M})$ are finite and the explicitly listed relations in (2.1) and (2.2) give presentations for these groups, respectively. If $g \geq 1$, then these presentations must contain at least one more relation.

Following [7], we label the vertices, edge-centers and face-centers of $\mathcal{M}$ with $\mathbf{0 ,} \mathbf{1}$ and 2 , respectively. They are called the geometric points of $\mathcal{M}$. As an automorphism preserves the geometric points, it follows that a mirror of a reflection of $\mathcal{M}$ passes through some geometric points of $\mathcal{M}$ and these geometric points form a periodic sequence. Since $S$ is compact, this sequence is finite and it is called the pattern of the mirror. As an example, consider the icosahedral map on the sphere, which has type $\{3,5\}$. Each reflection of this map fixes a mirror with pattern 010212010212 , which is abbreviated as $(\mathbf{0 1 0 2 1 2})^{2}$; see [7]. Each repeated part of a pattern is called a link, and the number of links is called the link index. So in this example, 010212 is a link and the link index is 2.

In [15], it has been shown that the pattern of a mirror is always obtained from one of the six links 01, 02, 12, 0102, 0212, 010212, and there cannot be more than three mirrors with different patterns on the same Riemann surface. (See Figures 1 and 2, which represent regular maps admitting two and three different patterns, respectively.) The following theorem expresses this idea and it can be deduced from [15].

Theorem 2.1. Let $\mathcal{M}$ be a regular map of type $\{m, n\}$ on a compact Riemann surface $S$ and let $M$ be a mirror of a reflection of $\mathcal{M}$. Then:

(i) If $m$ and $n$ are odd, then $M$ has pattern of the form $(\mathbf{0 1 0 2 1 2})^{\ell}$;

(ii) If $m$ is even and $n$ is odd, then $M$ has pattern of the form $(\mathbf{0 1 0 2})^{\ell_{1}}$ or $(\mathbf{1 2})^{\ell_{2}}$;

(iii) If $m$ is odd and $n$ is even, then $M$ has pattern of the form $(\mathbf{0 2 1 2})^{\ell_{1}}$ or $(\mathbf{0 1})^{\ell_{2}}$;

(iv) If $m$ and $n$ are even, then $M$ has pattern of the form $(01)^{\ell_{1}},(02)^{\ell_{2}}$ or $(02)^{\ell_{3}}$.

Here $\ell, \ell_{1}, \ell_{2}$ and $\ell_{3}$ are positive integers, which depend only on $\mathcal{M}$, not on $M$. Furthermore, $\ell_{i} s$ in different lines need not be equal.

Note that all the patterns listed in each part of Theorem 2.1 do occur. For example, in part (ii) the surface $S$ contains two classes of mirrors such that the mirrors in different classes have different patterns, namely $(\mathbf{0 1 0 2})^{\ell_{1}}$ and $(\mathbf{1 2})^{\ell_{2}}$. The same argument applies to all parts of Theorem 2.1.

Now let $\mathcal{M}$ be a regular map on a compact Riemann surface $S$ and let $M$ be a mirror of a reflection of $\mathcal{M}$. Suppose that $\ell$ is the link index of the pattern of $M$. If $\ell>2$, then there exist two orientation-preserving automorphisms of $\mathcal{M}$ of order $\ell$, which fix $M$ setwise and have no fixed points on $M$. They rotate $M$ in opposite directions and cyclically permute the links of the pattern of $M$. These automorphisms are inverses of each other and they are called the mirror automorphisms of $M$. Note that if $\ell=2$, then $M$ has a unique mirror automorphism. If $\ell=1$, then we assume that the mirror automorphism of $M$ is the identity. Associated to each pattern, there is a conjugacy class of mirror automorphisms such that the order of each mirror automorphism in this conjugacy class is equal to the link index of the pattern; see [15, Lemma 1]. In Table 1, for each pattern, a representative mirror automorphism is displayed in terms of the generators of $\mathrm{Aut}^{+}(\mathcal{M})$ in (2.2). Note that in the table, for each pattern, only one link is displayed. See [15] for details. 
Table 1: Patterns and mirror automorphisms.

\begin{tabular}{lll}
\hline Case & Link & Mirror automorphism \\
\hline \hline 1 & $\mathbf{0 1}$ & $Z^{\frac{n}{2}} X$ \\
2 & $\mathbf{0 2}$ & $Y^{\frac{m}{2}} Z^{\frac{n}{2}}$ \\
3 & $\mathbf{1 2}$ & $Y^{\frac{m}{2}} X$ \\
4 & $\mathbf{0 1 0 2}$ & $Z^{\frac{n+1}{2}} Y Z^{\frac{n+1}{2}} Y^{\frac{m}{2}}$ \\
5 & $\mathbf{0 2 1 2}$ & $Z^{\frac{n}{2}} Y^{\frac{m+1}{2}} Z Y^{\frac{m+1}{2}}$ \\
6 & $\mathbf{0 1 0 2 1 2}$ & $Y^{\frac{m+1}{2}} Z Y^{\frac{m+1}{2}} Z^{\frac{n+1}{2}} Y Z^{\frac{n+1}{2}}$ \\
\hline
\end{tabular}

\section{Number of mirrors}

From now on, $\|\mathcal{M}\|$ will denote the total number of mirrors fixed by the reflections of a regular map $\mathcal{M}$.

Theorem 3.1. Let $\mathcal{M}$ be a regular map of type $\{m, n\}$ on a compact Riemann surface, and let $\ell, \ell_{1}, \ell_{2}, \ell_{3}$ be as in Theorem 2.1. Then:

(i) If $m$ and $n$ are odd, then $\|\mathcal{M}\|=\frac{\left|\mathrm{Aut}^{+}(\mathcal{M})\right|}{2 \ell}$;

(ii) If $m$ and $n$ have different parities, then $\|\mathcal{M}\|=\frac{\left|\mathrm{Aut}^{+}(\mathcal{M})\right|}{2}\left(\frac{1}{\ell_{1}}+\frac{1}{\ell_{2}}\right)$;

(iii) If $m$ and $n$ are even, then $\|\mathcal{M}\|=\frac{\left|\mathrm{Aut}^{+}(\mathcal{M})\right|}{2}\left(\frac{1}{\ell_{1}}+\frac{1}{\ell_{2}}+\frac{1}{\ell_{3}}\right)$.

Proof. (i) By Theorem 2.1, every mirror of a reflection of $\mathcal{M}$ has pattern $(\mathbf{0 1 0 2 1 2})^{\ell}$. It is clear that each of these mirrors contains $\ell$ edges of $\mathcal{M}$. Since $\mathcal{M}$ has $\frac{\left|\mathrm{Aut}^{+}(\mathcal{M})\right|}{2}$ edges, we find that $\|\mathcal{M}\|=\frac{\left|\mathrm{Aut}^{+}(\mathcal{M})\right|}{2 \ell}$.

(ii) Suppose that $m$ is even and $n$ is odd. It follows from Theorem 2.1 that the pattern of a mirror of a reflection of $\mathcal{M}$ is either $(\mathbf{0 1 0 2})^{\ell_{1}}$ or $(\mathbf{1 2})^{\ell_{2}}$. It is known that $\mathcal{M}$ has $\frac{\left|\mathrm{Aut}^{+}(\mathcal{M})\right|}{m}$ faces and a mirror with pattern $(\mathbf{0 1 0 2})^{\ell_{1}}$ passes through the centers of $\ell_{1}$ faces of $\mathcal{M}$. Also, the number of mirrors with pattern $(\mathbf{0 1 0 2})^{\ell_{1}}$ passing through the center of a face $F$ of $\mathcal{M}$ is $m / 2$. (See Figure 1, where $m=6$ and $n=3$. The dashed lines denote the mirrors that have pattern $(\mathbf{0 1 0 2})^{\ell_{1}}$ and pass through the center of $F$.) Therefore, there are

$$
\frac{\left|\mathrm{Aut}^{+}(\mathcal{M})\right|}{m} \frac{1}{\ell_{1}} \frac{m}{2}=\frac{\left|\mathrm{Aut}^{+}(\mathcal{M})\right|}{2 \ell_{1}}
$$

mirrors with pattern $(0102)^{\ell_{1}}$. A similar argument shows that there are

$$
\frac{\left|\mathrm{Aut}^{+}(\mathcal{M})\right|}{m} \frac{1}{\ell_{2}} \frac{m}{2}=\frac{\left|\mathrm{Aut}^{+}(\mathcal{M})\right|}{2 \ell_{2}}
$$

mirrors with pattern $(\mathbf{1 2})^{\ell_{2}}$. As a result, we find that

$$
\|\mathcal{M}\|=\frac{\left|\mathrm{Aut}^{+}(\mathcal{M})\right|}{2}\left(\frac{1}{\ell_{1}}+\frac{1}{\ell_{2}}\right) .
$$




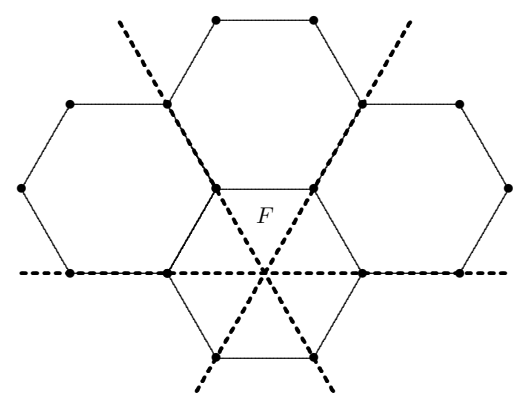

Figure 1: Mirrors with pattern $(\mathbf{0 1 0 2})^{\ell_{1}}$, passing through a face-center.

The case where $m$ is odd and $n$ is even is similar.

(iii) In this case, by Theorem 2.1 , the pattern of a mirror is either $(\mathbf{0 1})^{\ell_{1}},(\mathbf{1 2})^{\ell_{2}}$ or $(\mathbf{0 2})^{\ell_{3}}$. We know that $\mathcal{M}$ has $\frac{\left|\mathrm{Aut}^{+}(\mathcal{M})\right|}{n}$ vertices and a mirror with pattern $(\mathbf{0 1})^{\ell_{1}}$ passes through $\ell_{1}$ vertices of $\mathcal{M}$. Moreover, the number of mirrors with pattern $(\mathbf{0 1})^{\ell_{1}}$ passing through a vertex of $\mathcal{M}$ is $n / 2$. (See Figure 2, where $m=n=4$. The dashed lines denote the mirrors that have pattern $(\mathbf{0 1})^{\ell_{1}}$ and pass through a vertex $v$ of $\mathcal{M}$.) Thus, there are

$$
\frac{\left|\mathrm{Aut}^{+}(\mathcal{M})\right|}{n} \frac{1}{\ell_{1}} \frac{n}{2}=\frac{\left|\mathrm{Aut}^{+}(\mathcal{M})\right|}{2 \ell_{1}}
$$

mirrors with pattern $(\mathbf{0 1})^{\ell_{1}}$. Similar arguments show that there are $\frac{\left|\mathrm{Aut}^{+}(\mathcal{M})\right|}{2 \ell_{2}}$ mirrors with pattern $(\mathbf{1 2})^{\ell_{2}}$ and $\frac{\left|\mathrm{Aut}^{+}(\mathcal{M})\right|}{2 \ell_{3}}$ mirrors with pattern $(\mathbf{0 2})^{\ell_{3}}$. Consequently, we find that $\|\mathcal{M}\|=\frac{\left|\mathrm{Aut}^{+}(\mathcal{M})\right|}{2}\left(\frac{1}{\ell_{1}}+\frac{1}{\ell_{2}}+\frac{1}{\ell_{3}}\right)$.

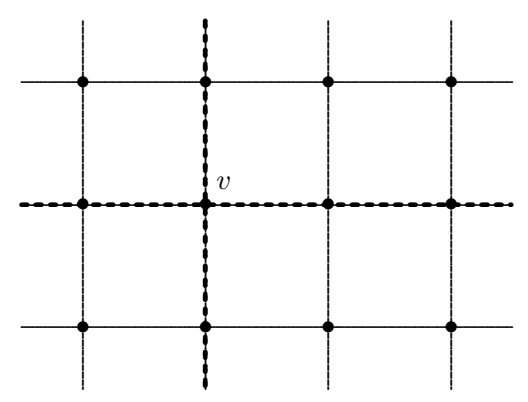

Figure 2: Mirrors with pattern $(\mathbf{0 1})^{\ell_{1}}$, passing through a vertex.

Note that if $\mathcal{M}$ is a reflexible regular map and if we are given a presentation for Aut $^{+}(\mathcal{M})$ as in (2.2), then we can easily determine the link indices by using Table 1 and MAGMA [1]. This is because the link indices are the orders of the mirror automorphisms (see [15, Lemma 1]), and the latter are explicitly known (see Table 1). Then by using Theorem 3.1 we can easily calculate $\|\mathcal{M}\|$. 
Example 3.2. Let $S$ be the Riemann surface of genus 7 admitting 504 conformal automorphisms. This surface is known as the Fricke-Macbeath surface; see [8, 14]. It is known that $S$ underlies a regular map $\mathcal{M}$ of type $\{3,7\}$, which is called the Fricke-Macbeath map. It follows from [15] that $\mathrm{Aut}^{+}(\mathcal{M})$ has a presentation

$$
\left\langle X, Y, Z \mid X^{2}=Y^{3}=Z^{7}=X Y Z=\left(Y^{2} Z Y^{2} Z^{4} Y Z^{4}\right)^{2}=1\right\rangle,
$$

and $Y^{2} Z Y^{2} Z^{4} Y Z^{4}$ is a mirror automorphism. Since this automorphism has order 2, by Theorem 2.1 every mirror on $S$ has pattern $(\mathbf{0 1 0 2 1 2})^{2}$. Thus, by using Theorem 3.1 we find that $\|\mathcal{M}\|=\frac{504}{4}=126$.

Remark 3.3. Let $\mathcal{M}$ be a regular map and $\mathcal{M}^{*}$ be its dual. Since the reflections of $\mathcal{M}$ and $\mathcal{M}^{*}$ coincide, the mirrors of $\mathcal{M}^{*}$ are the same as those of $\mathcal{M}$. So $\|\mathcal{M}\|=\left\|\mathcal{M}^{*}\right\|$.

Remark 3.4. Let $\mathcal{M}$ be a regular map on a compact Riemann surface $S$ and let $M$ be a mirror of a reflection of $\mathcal{M}$. If $\ell$ is the link index corresponding to the pattern of $M$, then the stabilizer of $M$ in $\operatorname{Aut}^{+}(\mathcal{M})$ is the dihedral group $D_{\ell}$. Here $D_{\ell}$ is generated by a mirror automorphism of $M$ and an involution fixing two antipodal points of $M$. Since $\mathrm{Aut}^{+}(\mathcal{M})$ is transitive on the mirrors with the same pattern, the orbit of $M$ consists of the mirrors on $S$ which have the same pattern as $M$. So by the Orbit-Stabilizer theorem, we find that there are $\left|\mathrm{Aut}^{+}(\mathcal{M})\right| / 2 \ell$ mirrors in the orbit of $M$. By Theorem 2.1, there are at most three orbits, and their sizes can be determined in the same way. Therefore, we obtain an alternative proof of Theorem 3.1.

\section{Lengths of mirrors}

Let $\mathcal{M}$ be a regular map of type $\{m, n\}$ on a compact Riemann surface $S$ of genus $g$ and let $M$ be a mirror of a reflection of $\mathcal{M}$. As pointed out in Section 2, $S$ can be divided into $\left|\operatorname{Aut}^{ \pm}(\mathcal{M})\right|(2, m, n)$-triangles and $M$ is a combination of the sides of $(2, m, n)$-triangles. Let $a, b$ and $c$ be the lengths of the sides of a $(2, m, n)$-triangle as indicated in Figure 3 . If $g>1$, then by using sine and cosine rules for hyperbolic triangles, we can calculate $a, b$ and $c$. So the length of $M$ can be calculated as described below.

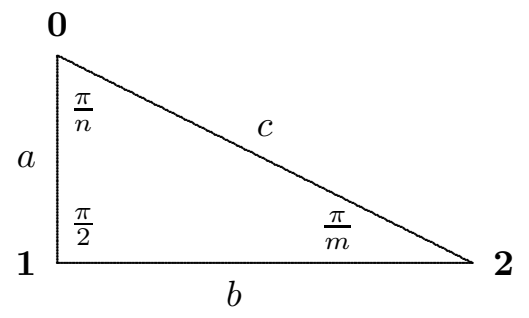

Figure 3: A $(2, m, n)$-triangle with side lengths $a, b, c$.

Let $m$ and $n$ be odd. Then $M$ will have pattern of the form $(\mathbf{0 1 0 2 1 2})^{\ell}$. Now every link corresponds to a segment of $M$, which has length $2(a+b+c)$. Thus, $M$ has length $2 \ell(a+b+c)$. Clearly, every mirror has the same length as $M$ in this case. If $m$ and $n$ have different parities, then there are two classes of mirrors on $S$. If $m$ and $n$ are both even, then there are three classes of mirrors on $S$. In both cases the mirrors in each class have the same length and pattern. In each case, the lengths of the mirrors can be calculated in the 
same way. Note that if $g=0$ or 1 , then it is not difficult to find the length of the mirrors explicitly. So we have the following result:

Theorem 4.1. Let $\mathcal{M}$ be a regular map of type $\{m, n\}$ on a compact Riemann surface $S$ and let the lengths of the sides of $a(2, m, n)$-triangle be $a, b$ and $c$ as indicated in Figure 3. Then the lengths of the mirrors of the reflections of $\mathcal{M}$ can be determined by the formulae in Table 2 , where $\ell, \ell_{1}, \ell_{2}$ and $\ell_{3}$ are the link indices and $\ell_{i} s$ in different lines need not be equal.

Table 2: Lengths of mirrors.

\begin{tabular}{lll}
\hline Case & Pattern & Length of mirror \\
\hline \hline$m$ and $n$ are odd & $(\mathbf{0 1 0 2 1 2})^{\ell}$ & $2 \ell(a+b+c)$ \\
$m$ odd $n$ even & $(\mathbf{0 1})^{\ell_{1}}$ & $2 \ell_{1} a$ \\
$m$ odd $n$ even & $(\mathbf{0 2 1 2})^{\ell_{2}}$ & $2 \ell_{2}(b+c)$ \\
$m$ even $n$ odd & $(\mathbf{1 2})^{\ell_{1}}$ & $2 \ell_{1} b$ \\
$m$ even $n$ odd & $(\mathbf{0 1 0 2})^{\ell_{2}}$ & $2 \ell_{2}(a+c)$ \\
$m$ and $n$ are even & $(\mathbf{0 1})^{\ell_{1}}$ & $2 \ell_{1} a$ \\
$m$ and $n$ are even & $(\mathbf{1 2})^{\ell_{2}}$ & $2 \ell_{2} b$ \\
$m$ and $n$ are even & $(\mathbf{0 2})^{\ell_{3}}$ & $2 \ell_{3} c$ \\
\hline
\end{tabular}

\section{Application to Hurwitz maps}

By a classical theorem of Hurwitz [11], a compact Riemann surface of genus $g>1$ has at most $84(g-1)$ conformal automorphisms. Any such surface $S=\mathbb{H} / \Lambda$ is called a Hurwitz surface, and in that case $\operatorname{Aut}^{+}(S)$ is called a Hurwitz group. It is known that if $S$ is a Hurwitz surface, then $\Lambda$ is normal in the ordinary triangle group $\Gamma[2,3,7]$. Thus, every Hurwitz surface underlies a regular map of type $\{3,7\}$, which is called a Hurwitz map. Furthermore, $\operatorname{Aut}^{+}(\mathcal{M})$ is isomorphic to $\operatorname{Aut}^{+}(S)$ and has a presentation of the form

$$
\left\langle X, Y, Z \mid X^{2}=Y^{3}=Z^{7}=X Y Z=\cdots=1\right\rangle .
$$

It has been shown by [13] that the upper bound in Hurwitz's theorem is attained for infinitely many values of the genus $g$. Thus, there exist infinitely many Hurwitz maps and surfaces. See [2, 3, 4, 5, 13] for further details.

Theorem 5.1. Let $\mathcal{M}$ be a Hurwitz map of genus $g$ and let $S$ be the underlying surface. Then $\|\mathcal{M}\| \leq 21(g-1)$, where equality holds if and only if $S$ is the Fricke-Macbeath surface.

Proof. Let $\ell$ be the link index of $\mathcal{M}$. By Theorem 3.1, we find that

$$
\|\mathcal{M}\|=\frac{\left|\mathrm{Aut}^{+}(\mathcal{M})\right|}{2 \ell}=\frac{84(g-1)}{2 \ell}=\frac{42(g-1)}{\ell} .
$$

It follows from [15, Theorem 5] that $\ell \geq 2$ and hence $\|\mathcal{M}\| \leq 21(g-1)$ and that equality holds if and only if $S$ is the Fricke-Macbeath surface. See also [9, Theorem 4.1]. 
It follows from Theorem 5.1 that if $\mathcal{M}$ is a Hurwitz map of genus $g$ with link index $\ell$, then $\|\mathcal{M}\|$ is bounded above by $21(g-1)$. When this upper bound is attained, $\ell=2$ and the underlying surface is the Fricke-Macbeath surface. However, $\|\mathcal{M}\|$ cannot have a lower bound in terms of $g$. This follows from the theorem below, which was given in [6].

Theorem 5.2. For every positive integer $n$, there exist Hurwitz maps with link indices $2 n$ and $3 n$. In particular, the link index of a Hurwitz map can be any even positive integer.

Let $L$ be the sum of the lengths of the sides a $(2,3,7)$-triangle. Then by using the sine and cosine rules for hyperbolic triangles we find that $L \simeq 1.4490747226$. It follows from Theorem 4.1 that the length of a mirror on a Hurwitz surface is $2 \ell L$, where $\ell$ is the link index. Also, the minimum possible length of a mirror on a Hurwitz surface is $4 L \simeq$ 5.7962988904 , and in that case the underlying surface is the Fricke-Macbeath surface; see [15, Theorem 6]. However, by Theorem 5.2, there is no upper bound on the lengths of mirrors on Hurwitz surfaces.

\section{References}

[1] W. Bosma, J. Cannon and C. Playoust, The Magma algebra system I: The user language, J. Symbolic Comput. 24 (1997), 235-265, doi:10.1006/jsco.1996.0125.

[2] M. Conder, The genus of compact Riemann surfaces with maximal automorphism group, $J$. Algebra 108 (1987), 204-247, doi:10.1016/0021-8693(87)90135-9.

[3] M. Conder, Maximal automorphism groups of symmetric Riemann surfaces with small genus, J. Algebra 114 (1988), 16-28, doi:10.1016/0021-8693(88)90208-6.

[4] M. Conder, Hurwitz groups: a brief survey, Bull. Amer. Math. Soc. (N. S.) 23 (1990), 359-370, doi:10.1090/s0273-0979-1990-15933-6.

[5] M. Conder, An update on Hurwitz groups, Groups Complex. Cryptol. 2 (2010), 35-49, doi: $10.1515 /$ gcc. 2010.002 .

[6] M. Conder and A. Melekoğlu, Link indices of Hurwitz maps, J. Algebra 490 (2017), 568-580, doi:10.1016/j.jalgebra.2017.08.001.

[7] H. S. M. Coxeter, Regular Polytopes, Dover Publications, New York, 3rd edition, 1973.

[8] R. Fricke, Ueber eine einfache Gruppe von 504 Operationen, Math. Ann. 52 (1899), 321-339, doi:10.1007/bf01476163.

[9] G. Gromadzki, On a Harnack-Natanzon theorem for the family of real forms of Riemann surfaces, J. Pure Appl. Algebra 121 (1997), 253-269, doi:10.1016/s0022-4049(96)00068-0.

[10] A. Harnack, Ueber die Vieltheiligkeit der ebenen algebraischen Curven, Math. Ann. 10 (1876), 189-198, doi:10.1007/bf01442458.

[11] A. Hurwitz, Ueber algebraische Gebilde mit eindeutigen Transformationen in sich, Math. Ann. 41 (1892), 403-442, doi:10.1007/bf01443420.

[12] G. A. Jones and D. Singerman, Theory of maps on orientable surfaces, Proc. London Math. Soc. 37 (1978), 273-307, doi:10.1112/plms/s3-37.2.273.

[13] A. M. Macbeath, On a theorem of Hurwitz, Proc. Glasgow Math. Assoc. 5 (1961), 90-96, doi:10.1017/s2040618500034365.

[14] A. M. Macbeath, On a curve of genus 7, Proc. London Math. Soc. 15 (1965), 527-542, doi: 10.1112/plms/s3-15.1.527.

[15] A. Melekoğlu and D. Singerman, The structure of mirrors on regular maps on Platonic surfaces, Geom. Dedicata 181 (2016), 239-256, doi:10.1007/s10711-015-0122-4. 